\title{
Geometric quality term for station-based total electron content estimation
}

\author{
Murat Selim Çepni๋, Erman Şentürk
}

University of Kocaeli, Department of Geomatic Engineering, Kocaeli, Turkey

\author{
Article history \\ Received June 5, 2015; accepted February 8, 2016. \\ Subject classification: \\ Ionosphere, TEC, R-TEC, Quality parameter.
}

\begin{abstract}
Global ionospheric maps (GIMs) are still commonly used to represent total electron content (TEC). However, a large number of permanent GPS receivers provide significant data for monitoring ionosphere. The GIMs supply accurate TEC results, although spherical models are not able to fit local ionospheric perturbations as a source of local receiver data. Therefore, the use of station-based TEC computation becomes more preferred and convenient. The station-based TEC approach is established on weighting averaging or modeling vertical TECs. Using this approach to reveal ionospheric activity depends on geometric distribution of ionospheric piercing points (IPP). The most effective parameter for examination of station-based TEC estimation is a function of relative geometry between receiver and satellite. In this study, a quality term has been used to define, for TEC estimation according to distribution of IPPs, similar to geometric dilution of precision (GDOP). The quality term has been described for the first time in this field and it was named as R-TEC (reliability of total electron content).
\end{abstract}

\section{Introduction}

Electromagnetic signals used in global navigation satellite systems (GNSS), travel through the ionosphere and interact with the ionospheric plasma, which causes propagation delays. The ionospheric range delay is positive for pseudorange measurements and negative for carrier phase measurements [Nohutcu et al. 2010]. Therefore, the carrier phase pseudoranges are measured to be too short and the code pseudoranges to be too long [Hofmann-Wellenhof et al. 1992]. Due to the fact that ionosphere is a dispersive medium; the ionospheric refraction depends on the signal frequency [Bergeot et al. 2014]. Thanks to dual frequency equipped receivers, ionospheric delay can be eliminated or estimated.

Electron density is a primary parameter used to describe ionosphere and ionospheric delaying effect on the GNSS signal which is a result of total electron content (TEC). The TEC is the total number of electrons in a vertical column of $1 \mathrm{~m}^{2}$ cross-section in the iono- sphere layer. Electron content within its path is measured in TEC unit (TECU) and 1 TECU is equal to $10^{16}$ electrons per square meter [Chauhan et al. 2011, Karia and Pathak 2011]. The observation of total number of free electrons along the ray path is derived from the frequencies $\mathrm{L}_{1}(1572.42 \mathrm{MHz})$ and $\mathrm{L}_{2}(1227.60 \mathrm{MHz})$ of the GPS that provide the relative ionospheric delay of electromagnetic waves, traveling through the medium [Saito et al. 1998, Ogunsua et al. 2014]. 10 TECU induces a delay of $5.4 \mathrm{~ns}(=1.6 \mathrm{~m})$ on the travel time of $\mathrm{L}_{1}$ signals, and a delay of $8.9 \mathrm{~ns}(=2.7 \mathrm{~m})$ on the travel time of $\mathrm{L}_{2}$ signals [Bergeot et al. 2014].

Ionospheric electron densities are very important parameters for studying its characteristics and effects on precise positioning, navigational applications, and electromagnetic signal transmissions [Panda et al. 2014]. The value of the TEC is used for making appropriate range corrections, as well as accounting for errors occurring in the range delays, to determine the effects of space weather-related events, such as geomagnetic storms and scintillations due to ionospheric irregularities [Rama Rao et al. 2006, Kumar et al. 2012]. Various scientific applications and services increasingly demand real-time information on the effects of ionosphere on the GPS signals. Several researches were carried out on the possibility of estimating the TEC, along the ray path, This was between a GPS satellite and ground-based receiver. This was used from the GPS receiver measurements [Mannucci et al. 1998, Ciraolo et al. 2007].

While traveling through ionosphere, the GNSS signals are exposed to delaying effects caused by electron density. Thorough relative time delay of $\mathrm{L}_{1}$ and $\mathrm{L}_{2}$ frequencies, the slant TEC (STEC) is calculated along the ray path from receiver to satellite. As the slant TEC is dependent upon the ray path, geometry through the ionosphere, it is required to calculate an equivalent vertical value of the TEC which is independent from the 
elevation of the ray path [Sharma et al. 2012]. The ionosphere is generally divided into several layers of varying thickness, according to electron density [Hargreaves 1992]. Used in the conventional TEC process, it is collapsed into a single layer. The single-layer model (SLM) assumes that the thin spherical shell located on a specified altitude, whereby the STEC is converted to the vertical TEC (VTEC). The slant TEC is projected to vertical TEC, using the thin shell model, assuming a height of $350 \mathrm{~km}$ [Weber et al. 1986]. The point where the signal penetrates the thin shell is called the ionospheric piercing point (IPP).

VTEC is computed at many IPPs from its time delays using dual frequency observations. Of course, the number of VTECs is equal to the number of received satellites for each epoch. A solution can be obtained from weighted averaging of all individual VTEC estimates. Weighted average is regarded as TEC for relevant epoch. Weighting algorithms are functions of relative position between satellite and receiver.

Station-based TEC represents ionospheric activity at receiver zenith. Preferential selection of signal paths closest to the receiver will provide a more realistic local TEC, over receiver location [Çepni et al. 2013]. Hence, it is desired that observations of satellites that are closer to zenith have more determinative results for stationbased TEC.

Depending on the positions of IPP and receiver location, relative geometry affects the quality of the TEC computation. Relative geometry between receiver and satellite has twin parameters: satellite elevation and azimuth angles. The satellite elevation angle defines the IPP distance from receiver zenith; the satellite azimuth angle determines local time difference between the receiver and the IPP. Depending on relative geometry terms, IPP distributions can bementioned as preferable or inadequate.

In this study, the intentions are to describe a term like dilution of precision (DOP) for station-based TEC estimations. The DOP is widely used as a criterion for selecting measurement units, since it characterizes the geometric effect on GPS positioning error. Quality of overall navigation, solutions can be determined by examining the dilution of precision [Wells et al. 1986]. It is well known that DOP comes in various flavors, including geometrical (GDOP), positional (PDOP), horizontal (HDOP), vertical (VDOP), and time (TDOP) [Langley 1999]. DOP concept should be used in different studies; e.g. Zirari examined the DOP in Wi-Fi-based positioning systems as signal strength dilution of precision $[\mathrm{Zi}-$ rari et al. 2009].

Main purpose of this study is to examine the influence of relative positioning between receiver and satellite on the efficiency of TEC estimation. It has to be emphasized that, the TEC computation with IPPs is distant and under a different local time to the receiver's zenith. It cannot illustrate to the ionospheric electron its density at the receiver location. Consequently, it has been described as a numeric parameter for the TEC estimation, regarding the ionospheric activity at the receiver location. This term was named as reliability of TEC (R-TEC).

\section{Methodology}

The geometry-free linear combination of GPS signals (L4), which is also called the ionospheric observable, is classically used for ionospheric researches and it is obtained by subtracting simultaneous pseudorange or carrier phase observations [Ciraolo et al. 2007].

$L_{4}=L_{1}-L_{2}=\lambda_{1} \phi_{1 k}^{i}-\lambda_{2} \phi_{2 k}^{i}$

$L_{4}=-a\left(\frac{1}{f_{1}^{2}}-\frac{1}{f_{2}^{2}}\right) S T E C+\lambda_{1} B_{1}-\lambda_{2} B_{2}+c D C B^{i}+c D C B_{k}$

$\frac{1}{K}=a\left(\frac{1}{f_{1}^{2}}-\frac{1}{f_{2}^{2}}\right)=9.524370$, and $\frac{1}{K}$ means kilometer to TECU. $D C B^{i}, D C B_{k}$ are satellite and receiver biases. Biases term have to be known or estimated. $\lambda_{1} B_{1}-\lambda_{2} B_{2}$ : the term is not equal to zero and it has to be estimated. $\mathrm{B}_{1}$ and $\mathrm{B}_{2}$ are constant bias due to initial phase ambiguity.

The slant TEC is estimated from (1) for each satellite that passes. STECs occur along the signal path in ionosphere and related to the length of the path. That is why STECs have to be converted to the vertical TECs, which are comparable in terms of value for electron density.

Although the ionosphere consists of several layers on processing, the conversionof the STECs is assumed as a single layer. The single-layer model is used, whereby the maximum-density free electrons are accepted as being inside an area with infinitesimal thickness [Hugentobler et al. 2001, Alizadeh et al. 2011]. Briefly, VTECs are obtained by means of thin shell sphere. Figure 1 illustrates geometry of SLM.

$$
V T E C=S T E C_{1} \sqrt{1-\left[\frac{R_{E}}{R_{E}+h_{m}}(\sin \varepsilon)\right]^{2}} .
$$

Equation (2) is a well-known mapping function for the calculation of electron density, at vertical direction of the IPP. Thus, the VTECs are associated with the IPP. To describe the ionospheric electron density over the station, the TEC is computed from weighted average of the VTECs. Although local ionospheric modeling can also be used, weighting average is confidential for station-wise TEC computation. Conventionally univariate functions have been defined based on satellite elevation angle, for weighting. However in this study, it 


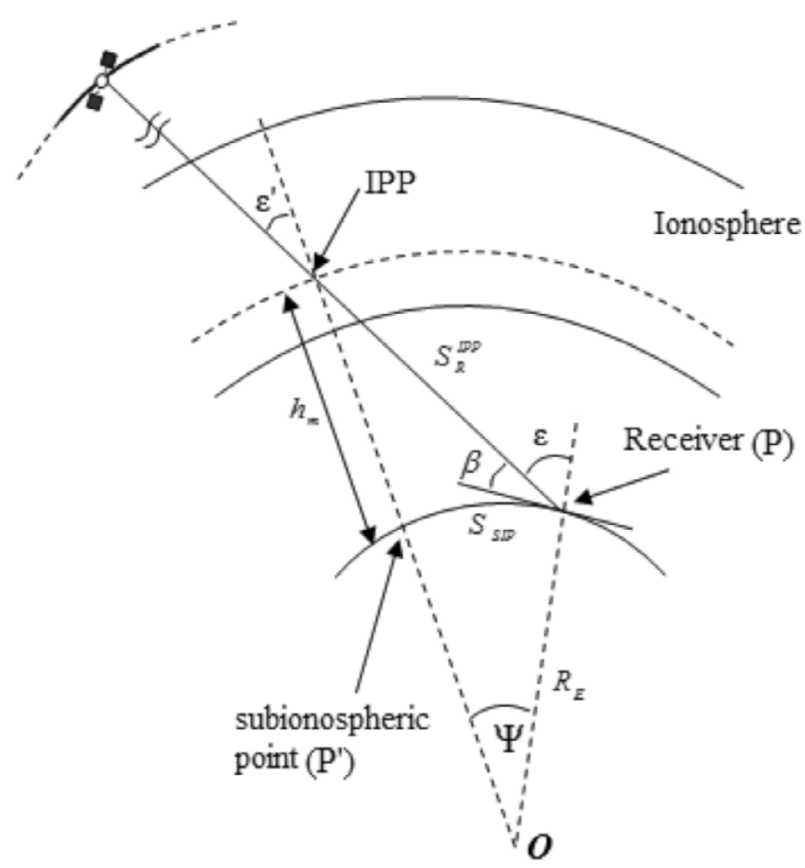

Figure 1. Single-layer model [Çepni et al. 2013].

has been proposed using of bivariate functions, composed of satellite elevation $(\varepsilon)$ and azimuth angles $(\alpha)$.

$$
T E C=\left.\frac{\sum_{i=1}^{N} W_{i}(\varepsilon, \alpha) V T E C_{i}}{\sum_{i=1}^{N} W_{i}(\varepsilon, \alpha)}\right|_{T_{1}} ^{T_{2}}
$$

where $T_{1}-T_{2}$ is time lapse interval.

As the users can choose the time interval, they can also use the default interval from the observation file. In this case the TEC is computed, e.g., 43,200 times for $2 \mathrm{~s}$ time intervals and 2880 times for $30 \mathrm{~s}$ time intervals within a day.

Due to the IPP associated processing, if the IPPs are away from receiver zenith, the weighted averaging or local modeling may not represent electron density at station's location. Distribution of the IPPs is decisive on the station dependent TEC. In other words, relative geometries between the receiver and the satellites affect TEC estimation quality.

Relative position of the receiver and the IPP has been comprised by satellite elevation and azimuth angles. The projection of ionosphere piercing point on the Earth is called the sub-ionosphere point (SIP) [Çepni et al. 2013]. Instead of the radius vector between the IPP and the receiver, the distance between SIP and receiver $\left(S_{S I P}\right)$ has to be taken into account. Hence in quality terms, variables which have to be explained are elevation and azimuth angles. As the satellite elevation angle describes the distance between the SIP and the receiver, the satellite azimuth angle characterizes longitudinal components of this distance.

The distance between the SIP and the receiver can be expressed as in (4) with respect to elevation angle. Additionally, Table 1 is arranged to show distances calculated from (4).

$$
S_{\mathrm{SIP}} \cong 2 R_{E} \arcsin \left(\frac{h_{m} \cot \varepsilon}{2 R_{E}}\right)
$$

where $\varepsilon, R_{E}$ and $h_{m}$ symbolize the satellite elevation angle, the radius of the Earth and the ionosphere height, respectively. $R_{E}$ and $h_{m}$ are accepted as $6378 \mathrm{~km}$ and $450 \mathrm{~km}$.

Low elevation angles may cause SIP to be so far from the receiver that the VTEC bears little resemblance to local conditions over the station. As seen in Table 1, the distance can be over $1000 \mathrm{~km}$ where there are lower elevation angles and the most frequently observed distances are between $200 \mathrm{~km}$ and $800 \mathrm{~km}$. If the $\mathrm{S}_{\mathrm{SIP}}$ distance exceeds a few hundred kilometers, VTEC may not represent receiver location. Thus if the elevation angle is low, representing ability of VTEC is interpreted as weak.

Satellite azimuth angle identifies the direction of $\mathrm{S}_{\mathrm{SIP}}$. So, time difference between the SIP and the receiver is determined by the elevation and azimuth angles. Local time effect may correspond to significant gaps on the estimated VTEC, due to daily TEC cycles, depending on the longitudinal component of $S_{\text {SIP }}$ [Şentürk 2014].

Diurnal variation of TEC is a characteristic in accordance to the rotation of the Sun and curve of daily TEC looks like a parabola reaching maximum at local noon. Local time of SIP indicates a shifting on diurnal TEC cycle from receiver location. Offsets for the e.g. with a 15 min time difference may reach to 2 TECU. Consequently, minimum longitudinal distance guarantees a better situation in terms of a local time parameter.

Longitudinal difference can be computed with respect to azimuth $(\alpha)$ and elevation angles $(\varepsilon)$. Figure 2 shows longitude difference as depending to elevation and azimuth angles.

$$
\Delta L=\frac{S_{\mathrm{SIP}} \sin \alpha}{R_{E} \cos \varphi_{M} \rho^{\circ}}
$$

where $\varphi_{M}$ means latitude for $\mathrm{S}_{\mathrm{SIP}} ; \rho^{\circ}=180^{\circ} / \pi$.

\begin{tabular}{cccccccccc}
\hline$\varepsilon^{0}$ & 10 & 20 & 30 & 40 & 50 & 60 & 70 & 80 & 90 \\
$\mathrm{~S}_{\mathrm{SIP}} \mathrm{km}$ & 2569 & 1238 & 779 & 536 & 377 & 259 & 163 & 79 & 0 \\
\hline
\end{tabular}

Table 1. Distances of $S_{\text {SIP }}$ according to elevation angles. 


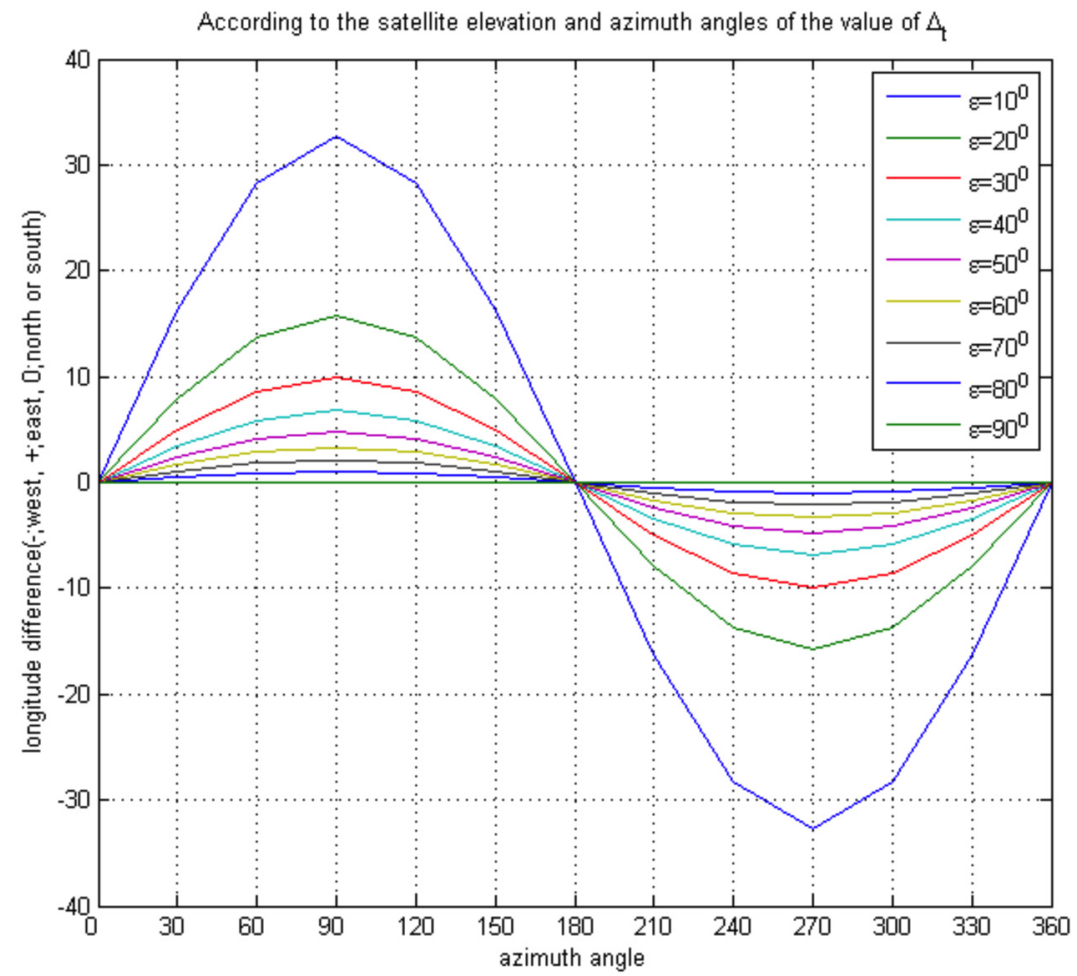

Figure 2. Longitudinal difference according to elevation and azimuth angles.

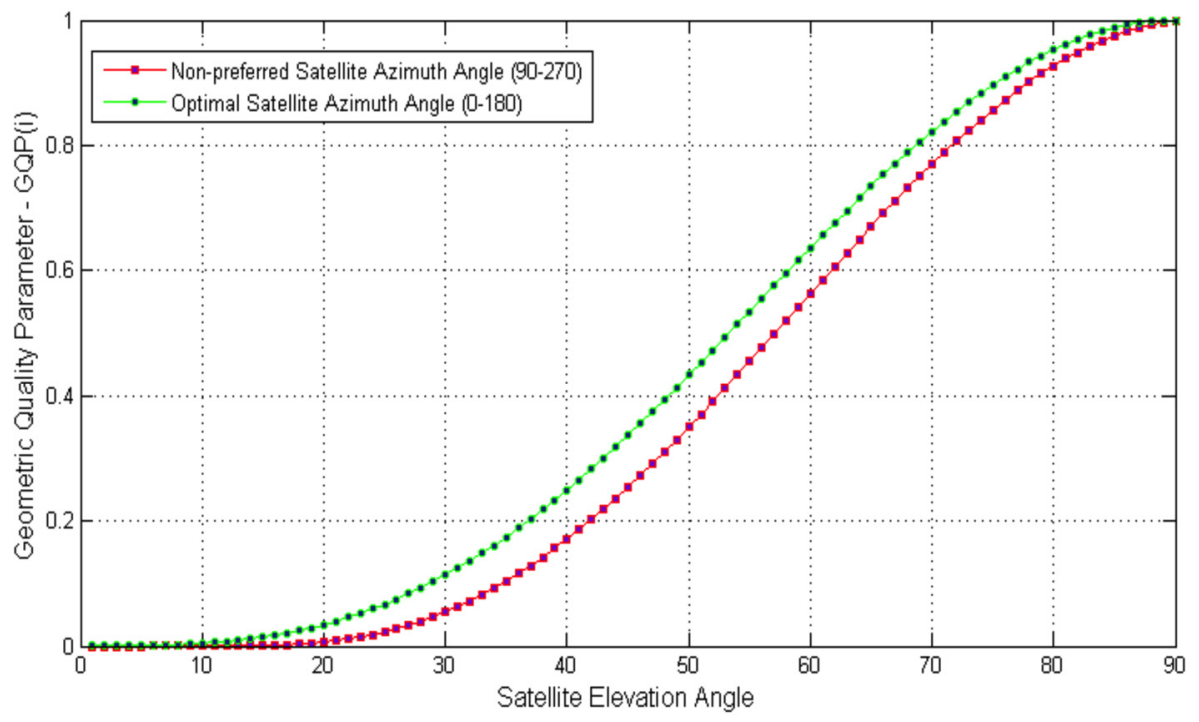

Figure 3. GQP graphic according to elevation and azimuth (extreme) angles.

After describing relative geometry components and their influences on the TEC estimation, the geometric quality parameter (GQP) can be defined. Mathematical expression of the GQP was developed empirically as a function of satellite elevation and azimuth angles.

$$
\mathrm{GQP}^{(\mathrm{i})}=\left(\sin \varepsilon_{n}^{(\mathrm{i})}\right)^{\pi} e^{-\left(\pi \Delta_{L n}^{(i)} / \varepsilon_{n}^{(i)}\right)}
$$

Equation (6) has been defined for all observed satellites at each epoch. Indices of $i$ and $n$ indicate the satellite number and epoch. Composition of GQP is based on proper usage of variables. Sinus function is widely used with the elevation angles in weighting the algorithms and the exponential function is well-done and complements the local time effect. Prepotency of two variables has been taken into consideration. The elevation angle must be more effective on the GQP and the effect of azimuth angle should increase a little relatively to how elevation angle should move away from zenith. It has been seen that the behavior of Equation (6) ensures geometric relationships presented by variables. Figure 3 outlines the behavior of GQP function.

The $\mathrm{GQP}^{(\mathrm{i})}$ has values at a [0-1] range. When the value is closer to 1, the GQP expresses ideal relative po- 

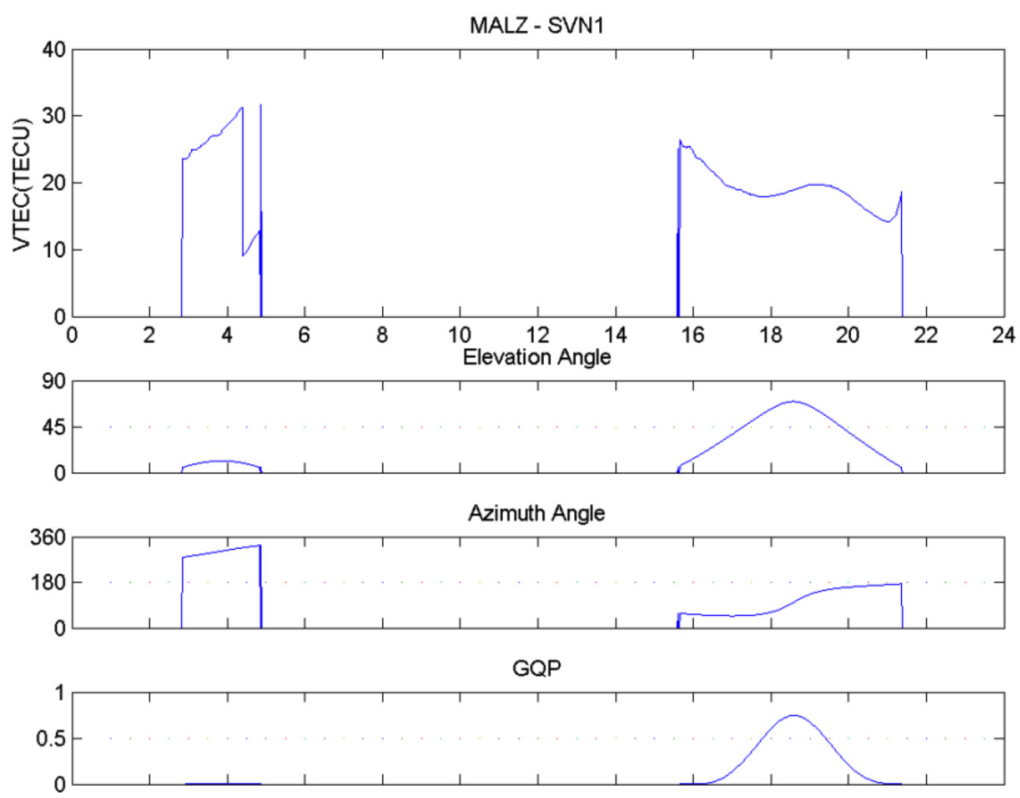

Figure 4. Plots for SVN1 and MALZ station on October 23, 2011.

sitioning for the satellite and receiver. In case that the value descends to 0 , the GQP means its insufficient IPP position cannot represent electron content at the receiver location.

The GQP ${ }^{(i)}$ is associated with the IPP and also the VTEC. Because of the fact that TEC is computed from VTECs via weighting average or local modeling, geometric quality terms for the station should be evaluated sum or average of the GQP ${ }^{(i)}$. In other words, individuals representing the ability of each observation and determines quality of estimation for the receiver. Thereby, statistical average of the GQPs was accepted as geometric quality term: reliability of TEC (R-TEC). It has to be noted that reliability was used in terms of sufficient relative geometry for TEC estimation.

$$
\mathrm{R}-\mathrm{TEC}=\sqrt{\left(\mathrm{GQP}^{(1)}\right)^{2}+\left(\mathrm{GQP}^{(2)}\right)^{2}+\ldots+\left(\mathrm{GQP}^{(\mathrm{i})}\right)^{2}}
$$

where $i$ denotes observed satellite.

Equation (7) reminds expression of dilution of precision (DOP). Both conceptual and mathematical, R-TEC has resemblance to GDOP. R-TEC can be computed at each epoch or time interval for station-based TEC estimation. Examination of distribution of R-TEC and discussion on its thresholds are in next section.

\section{Results and discussions}

In this study, two types of practice were performed. The first implementation aimed to introduce GQP quality term, and the $30 \mathrm{~s}$ resolution data on October 23, 2011, was used. The data were collected from MALZ and MURA stations which belong to the Continuously operating reference station - Turkey (CORS-TR) network. The second implementation intended to show samples from low, middle and high latitudes. The data, also $30 \mathrm{~s}$ resolution, were collected at three IGS stations on March 1, 2012. The VTECs have been processed from observation files initially and they were received as input for implementations. Afterward, geometric quality parameters GQP were computed for all observations from (6).

Figure 4 was arranged for sampling of implementations and it has drawings at MALZ station for SVN1 (svn1: satellite identification). The VTEC, elevation angle, azimuth angle and the GQP variations were drawn all day long. Relationships between relative geometry and the GQP ${ }^{1}$ were monitored in Figure 4. Also, VTEC and its geometric quality parameter can be seen together. It is suggested that VTEC graphics should include GQP bar, like the first and the last rows of Figure 4.

When Figure 4 is examined, it can be seen that the reliability of the VTECs is very low at 03-05 UT depending on relative geometry of observed satellites. This is because the elevation and azimuth angles are about $15^{\circ}$ and $300^{\circ}$, the VTECs have less quality. The $\mathrm{GQP}^{1}$ has no values at this period. Owed to GQP, users can be aware of unusable observations at this period. It is suggested that the VTEC is not taken into account in averaging, due to its low GQP.

Maximum $\mathrm{GQP}^{1}$ reading of the day $(\approx 0.80)$ appears just before $19 \mathrm{UT}$ due to its approximately $70^{\circ}$ in elevation. Azimuth has non-extreme case at this time. Geometric quality parameter indicates that the IPP ${ }^{1}$ associated with the VTEC can exemplify ionospheric plasma at the receiver location at 17:45-19:15 UT sufficiently. Out of this time interval, $\mathrm{SVN}^{1}$ observations are not suitable to define VTEC for this station. 


\begin{tabular}{|c|c|c|c|c|c|c|c|c|}
\hline$\varepsilon^{0}$ & 12 & 29 & 52 & 23 & 35 & 53 & 21 & 22 \\
\hline$\alpha^{0}$ & 192 & 228 & 46 & 81 & 126 & 309 & 167 & 287 \\
\hline $\mathbf{G Q P}^{(\mathbf{i})}$ & 0.002 & 0.049 & 0.385 & 0.009 & 0.104 & 0.406 & 0.025 & 0.008 \\
\hline \multirow[t]{2}{*}{ R-TEC } & 0.5716 & & & & & & & \\
\hline & & & & & & & & \\
\hline$\varepsilon^{0}$ & 61 & 69 & 32 & 11 & 21 & 52 & 35 & 39 \\
\hline$\alpha^{0}$ & 87 & 112 & 197 & 321 & 120 & 311 & 48 & 272 \\
\hline $\mathbf{G Q P}^{(\mathbf{i})}$ & 0.554 & 0.743 & 0.105 & 0.000 & 0.006 & 0.379 & 0.104 & 0.133 \\
\hline R-TEC & 1.3868 & & & & & & & \\
\hline
\end{tabular}

Table 2. (a) Minimum R-TEC of October 23, 2011 - Epoch: 05:07:30; (b) maximum R-TEC on October 23, 2011 - Epoch: 14:26:00.
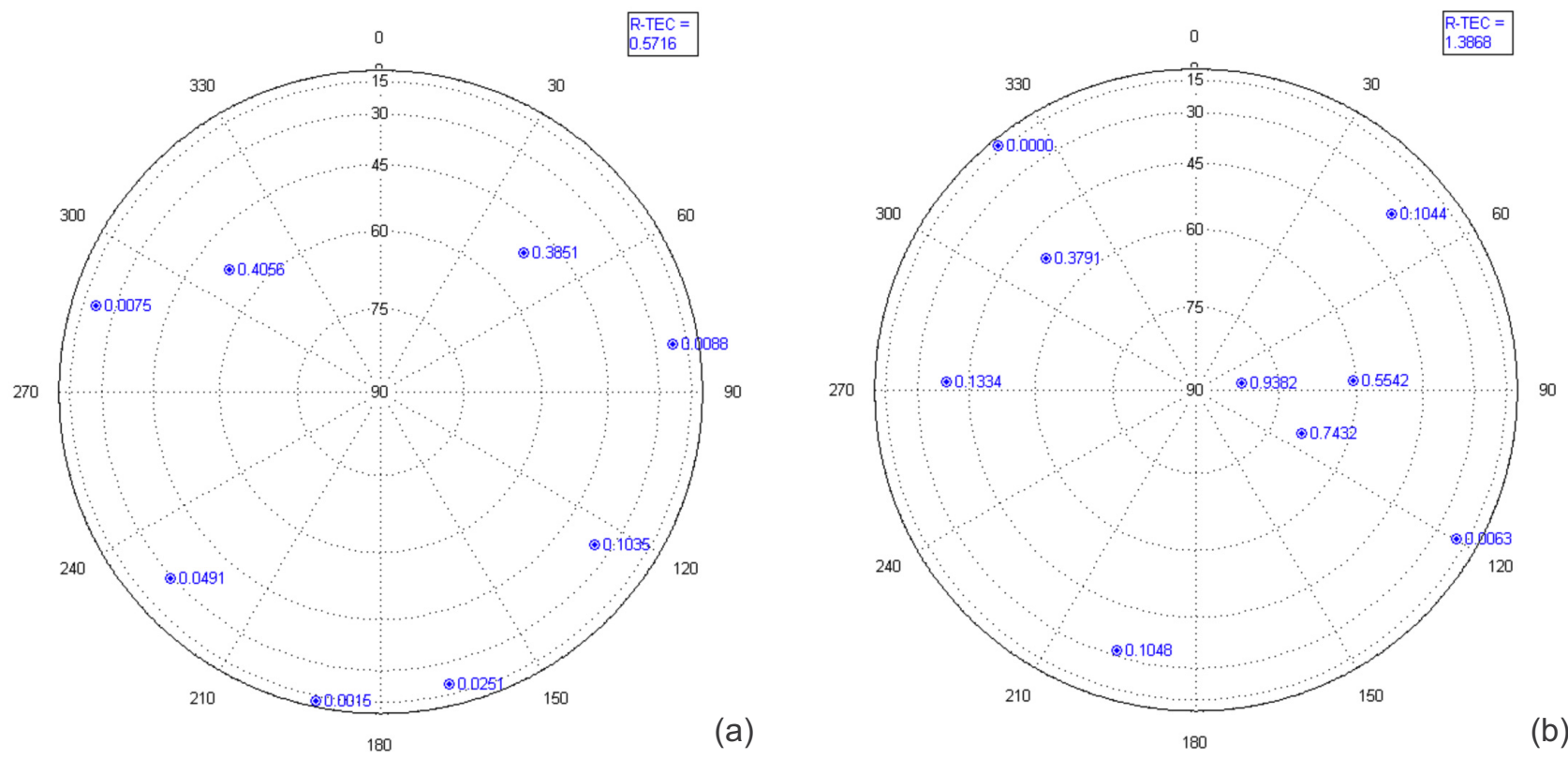

Figure 5. (a) Satellite chart for the minimum R-TEC; (b) Satellite chart for the maximum R-TEC.

Since the TEC is obtained from the VTECs, geometric quality term R-TEC has been described as the statistical average of geometric observation quality parameters of the VTECs $\left(\mathrm{GQP}^{(\mathrm{i})}\right)$. Figure 6 is the graphic image of daily R-TEC for the MALZ station. Tables 2a and $2 \mathrm{~b}$ figure out minimum and maximum R-TECs of the session day. The tables include quality parameter and its variables to make comparisons. Also, Figures $5 \mathrm{a}$ and $5 \mathrm{~b}$ have been charted in order to demonstrate IPP distributions for minimum and maximum rates of the R-TEC.

The effect of the elevation and azimuth angles on the GQP can be viewed once again from the tables. Due to the preferred azimuth angle, GQP is better than the others even when elevation angles are slightly lower, such as in Table 2a, columns 4, 7 and 8. When the tables are examined, correlations between the relative geometry and the quality parameter can be seen clearly, e.g., epoch of minimum R-TEC has no obser- vation over $55^{\circ}$ elevation angle.

The track of R-TEC throughout the day was plotted in Figure 6. The graphic depicts information to review its quality. The geometric reliability term appeared between 0.57 and 1.39 , it exceeds " 1 " equals approximately to 70 percent. Eventually, Figure 6 exhibits the final product of this study and it needs special examination. Moreover, the examination of reliability terms is required to be analyzed by outputs.

In order to reveal the distribution of the R-TEC, 15 days of data was processed. Due to repeated satellite constellation, trial data has been considered enough. The data belongs to the MALZ station in October 2011. The data consist of 43,200 epochs in total, and 6600 epochs were unable to process. The distribution can be seen in Figure 7.

It has been examined that Equation (7) produces values mostly $0.1-1.4$ interval and resemble the asymmetric Gaussian distribution. The skewness of distri- 


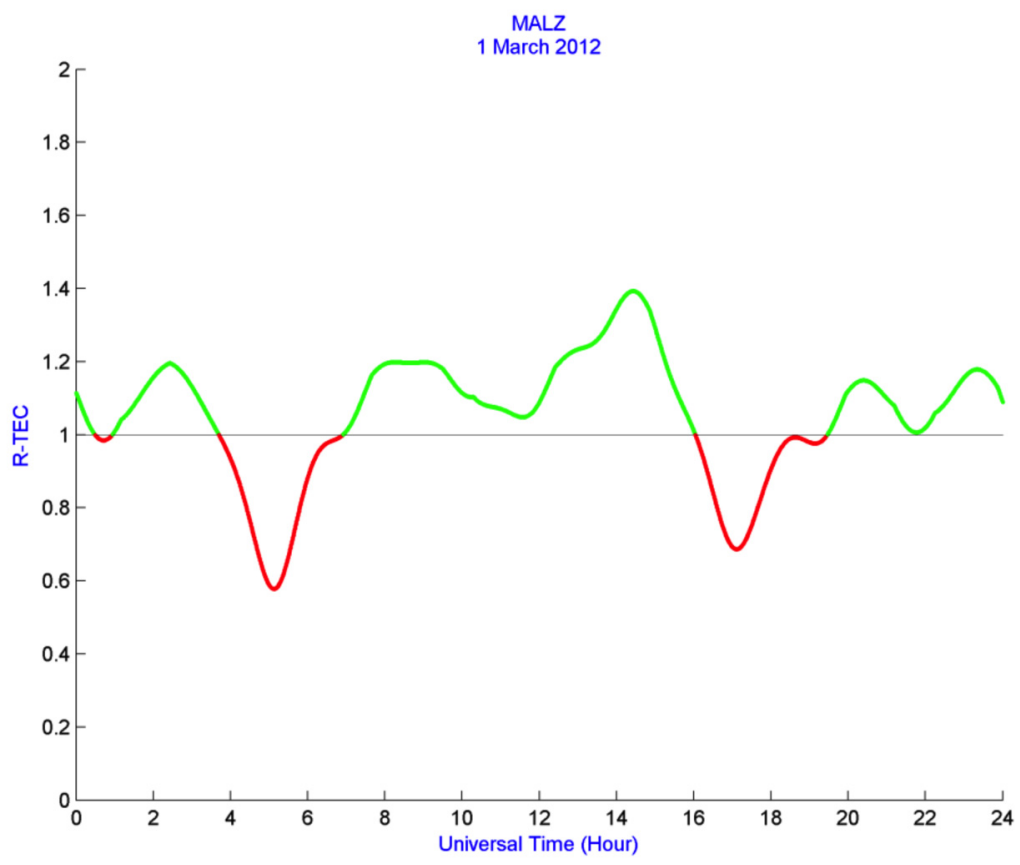

Figure 6. Plot of the daily R-TEC for the MALZ station on October 23, 2011.

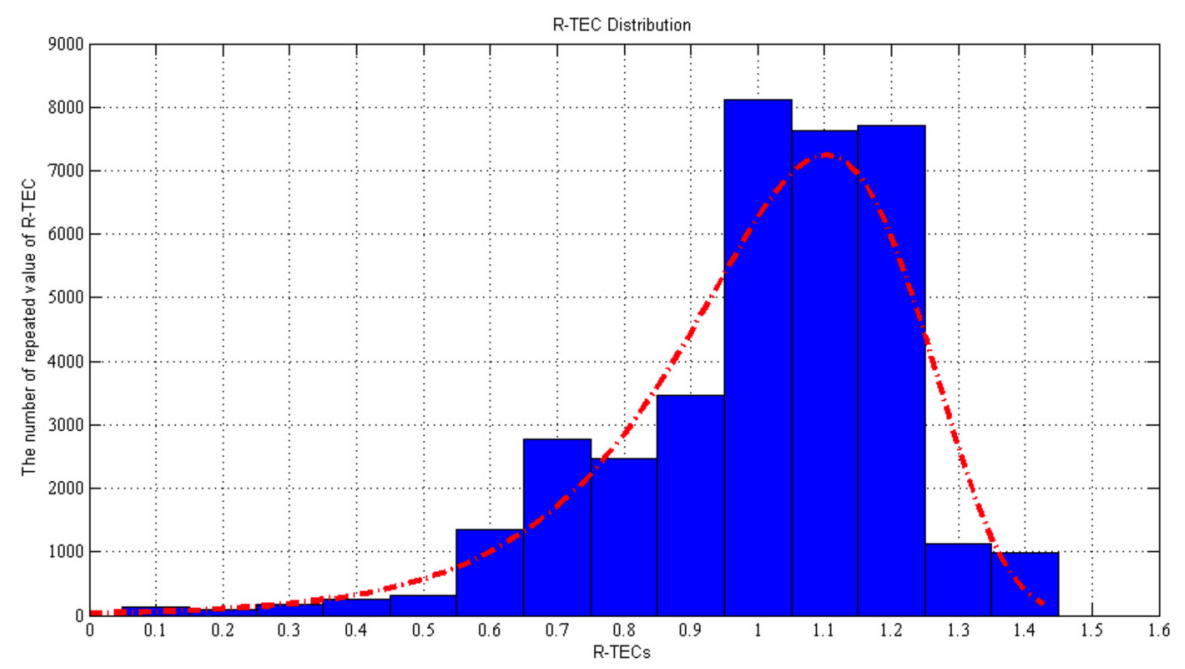

Figure 7. Distribution of the R-TEC values.

bution can be distinguished easily from bell curve on left (negative) skew. Negative skewness for quality terms means convergence to better cases and it points out that it is likely to provide sufficient reliability. Then the graphic information of the R-TEC distribution can be evaluated as realistic.

Statistical parameters are also shown in Figure 7. Mean, median and mode of distribution are very close to each other and all indicate 1 . Without having a threshold in statistics, values which are less than 0.6 and more than 1.2 may be interpreted as extreme, by accumulation of the values. In fact, determination of critical values for reliability terms is primarily significant. To qualify the TEC estimation, the critical value has to be determined.
As well known, about $68 \%$ of values drawn from a normal distribution are within one standard deviation away from the mean.

$$
P(\mu-\sigma<X<\mu+\sigma)=0.6826 \text {. }
$$

With a great similarity, $69 \%$ of the R-TEC values are over the 1 in this implementation.

$$
1-F(0.9)=0.6980
$$

Range equality in Equations (8) and (9) should be considered as proof of the R-TEC distribution limit value. It can be stated that rate 1 is the critical value, and, if the R-TEC is greater than or equal to 1 , the sta- 


\begin{tabular}{cccc} 
Id & Location & Lon $(\mathbf{E})^{\circ}$ & Lat $(\mathbf{N})^{\circ}$ \\
\hline reso & Resolute/Canada & 265.1067 & 74.6908 \\
ankr & Ankara/Turkey & 32.7586 & 39.8875 \\
riop & Riobamba/Ecuador & 281.3489 & -1.6506 \\
\hline
\end{tabular}

Table 3. Information of IGS stations used for interpreting at different latitudes.

tion dependent TEC estimation can be assumed to have a reliable geometry. In addition to confidence intervals in Equations (8) and (9), distributions parameters (mean, median, mode) confirm rate 1 with respect to the accumulation of the data set.

It must be stated that the R-TEC is an indicator for illustrating the ability of electron density at the receiver location. In case the R-TEC is less than 1, the IPP positions may not be competent in the estimation of the TEC for the receiver location.

The behavior of GQP at low and high latitude is examined in general. Implementation was realized at three IGS stations located at low, mid and high latitudes for data samples on March 1, 2012. The station informations are given in Table 3.

RESO station is located at Cornwallis Island at $\sim 75^{\circ}$ latitude. Because of the GPS constellation, it is expected that this station has bad visible satellite geometry for VTEC computation. RIOP station, at Ecuador, is a sample at low latitude for GQP, and ANKR station's data is used as mid-latitude sample.

Figure 8 shows efficiency of visible satellite geometry on reliable of V-TEC. At RESO station, R-TEC values are lower than other's, as expected, due to to orbital deficiency. According to position of station and time, relative geometry between receiver and observed satellites may not be sufficient any time. Thus, implementations at high and low latitudes point out signification of quality term R-TEC.

\section{Conclusion}

The relative geometry between the receiver and the satellites has considerable importance on the station-based TEC process. Certainly, accomplishment of estimation is influenced by this geometry. In this study, it has been aimed to compose a criterion depending on relative geometry components: satellite elevation and azimuth angles.

The effects of the elevation and the azimuth angles on the TEC computation were explained in detail in the previous sections. The impact factor of the IPP positions has been defined as the geometric quality parameters (GQPs). The GQP was expressed mathematically as in (6) by experimental study on composition of vari-
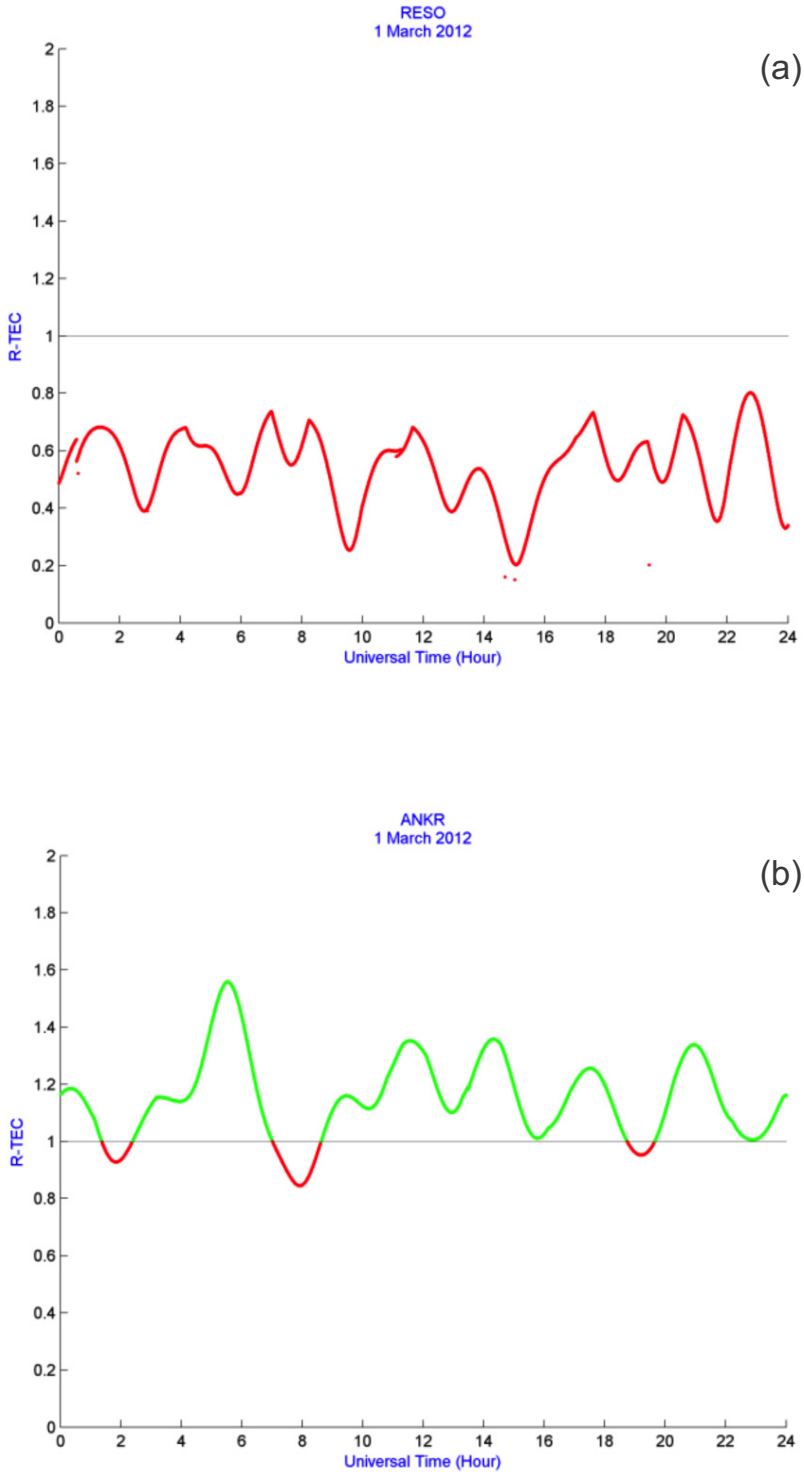

(b)

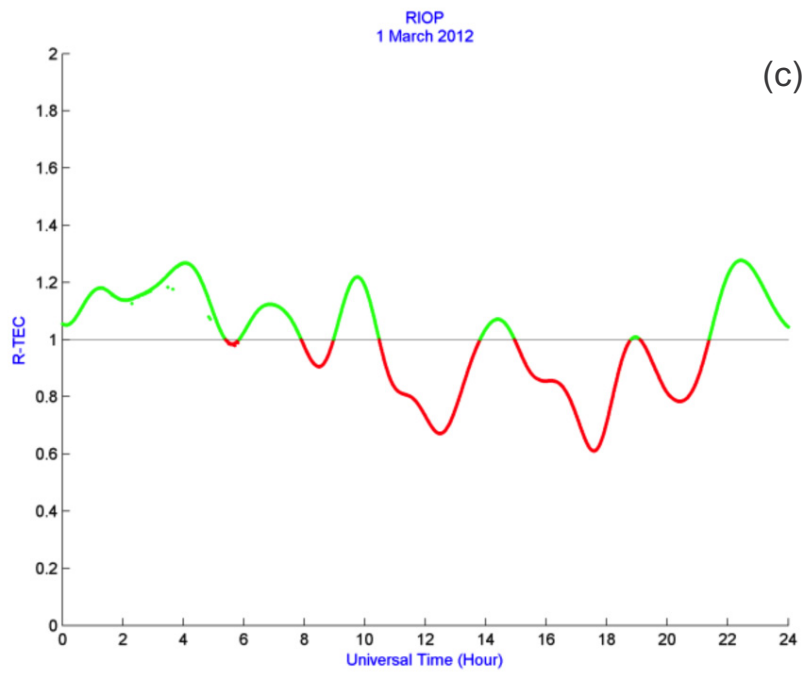

Figure 8. R-TEC values of IGS stations on March 1, 2012. (a) RTEC values at RESO station located at low-latitude; (b) R-TEC values at ANKR station located at middle-latitude; (c) R-TEC values at RIOP station located at high-latitude. 
ables. The GQP introduces representing ability to the ionospheric activity at receiver location by values within [0-1]. 0 and 1 demonstrate extremely inadequate and most sufficient IPP positions, respectively. The GQP approaches 1 for observations closer to the receiver zenith, while it decreases to zero for observations away from zenith in case of a local time difference.

Mathematical expression of the GQP has satisfactory form to cover desired features. It can be said that GQP criteria are able to present quality for the VTEC process. In addition to the quality factor, Equation (6) can be used as stochastic model for regional modeling or averaging of the VTECs. Actually, regional modeling on the CORS networks may require suitable weighting function just like the GQP.

The quality of station-based TEC process has been described as a statistical average of GQPs and it was named as reliability of TEC. It has to be emphasized that reliability should be evaluated in terms of appropriate relative geometry.

The geometric quality term (R-TEC) was formulated as in (7) and the R-TEC varies within 0.1-1.4 range. The distribution of the R-TEC values has been examined in order to detect a threshold. As a result of the accumulation analyze of the data, 1 has been determined as a limit for qualification. The R-TEC over 1 can be accepted as effective IPP geometry to estimate TEC. Nevertheless, it has to be noticed that R-TEC is always below 1 threshold at high latitudes.

The GQP and the R-TEC validate both averaging and modeling of the VTECs. Independent from method, geometric quality terms qualify the TEC estimations related to the IPP's relative locations.

Even if TEC is obtained via weighted average, it is mentioned from a simple modeling according to the positions of the IPP. The R-TEC informs us about relative positions of the IPP that are referenced in a form of modeling. It is possible to conceive that in case of a high enough R-TEC, the modeling is implemented in the small and local area. On the contrary, if the R-TEC is less than a necessary value, the IPPs are far away from the receiver and the modeling is realized at a wide region. The smaller the geometric quality term gets, the larger the area becomes. Thus the station dependent TEC estimation becomes similar to spherical models.

Due to the fact that the GPS constellation provides observation facility, it is not expected to lower the RTEC for a long time. However, Figure 6 shows that this period may be over $3 \mathrm{~h}$ twice a day. It is beneficial that the users should be aware of non-local TEC estimation along these periods. It is suggested to consult global ionospheric maps (GIMs) at such sessions.

\section{References}

Alizadeh, M., H. Schuh, S. Todorova and M. Schmidt (2011). Global ionospheric maps of VTEC from GNSS, satellite altimetry and FORMOSAT-3 / COSMIC data, Journal of Geodesy, 85, 975-987.

Bergeot, N., J.M. Chevalier, C. Bruyninx, E. Pottiaux, W. Aerts, Q. Baire, J. Legrand, P. Defraigne and W. Huang (2014). Near real-time ionospheric monitoring over Europe at the Royal Observatory of Belgium using GNSS data, Journal of Space Weather and Space Climate; doi:10.1051/swsc/2014028.

Çepni, M.S., L.V. Poots and J.B. Miima (2013). High Resolution Station-based Diurnal Ionospheric Total Electron Content (TEC) from Dual-frequency GPS observations, Space Weather, 11, 520-528.

Chauhan, V., O.P. Singh and B. Singh (2011).Diurnal and seasonal variation of GPS-TEC suring a low solar activity period as observed at a low altitude station Agra, Indian Journal of Radio and Space Physics, 40, 26-36.

Ciraolo, L., F. Azpilicueta, C. Brunini, A. Meza and S.M. Radicella (2007). Calibration errors on experimental Slant Total Electron Content (TEC) determined with GPS, Journal of Geodesy, 81, 111-120.

Hargreaves, J.K. (1992). The Solar Terrestrial Environment, Cambridge Atmospheric and Space Science Series, Cambridge, U.K.

Hofmann-Wellenhof, B., H. Lichtenegger and J. Collins (1992). Global Positioning System, Theory and Practice, Springer, Berlin.

Hugentobler, U., S. Schaer and P. Fridezi (2001).Bernese GPS Software Version 4.2., Astronomical Institute, University of Berne, Sweden.

Karia, S.P., and K.N. Pathak (2011). GPS based TEC measurements for a period August 2008-December 2009 near the northern crest of Indian equatorial ionospheric anomaly region, Journal of Earth System Science, 120, 851-858.

Kumar, S., S. Priyadarshi, S.G. Krishna and A.K. Singh (2012). GPS-TEC variations during low solar activity period (2007-2009) at Indian low latitude stations, Astrophysics and Space Science, 339, 165-178.

Langley, R.B. (1999). Dilution of Precision, GPS World, 52-59.

Mannucci, A.J., B.D. Wilson, D.N. Yuan, C.H. Ho, U.J. Lindqwister and T.F. Runge (1998). A global mapping technique for GPS-derived ionospheric total electron content measurements, Radio Science, 33, 565-582.

Nohutcu, M., M.O. Karslioglu and M. Schmidt (2010). B-spline modeling of VTEC over Turkey using GPS observations, Journal of Atmospheric and SolarTerrestrial Physics, 72, 617-624. 
Ogunsua, B.O., J.A. Laoye, I.A. Fuwape and A.B. Rabiu (2014). The comparative study of chaoticity and dynamical complexity of the low-latitude ionosphere, over Nigeria, during quiet and disturbed days, Nonlinear Processes in Geophysics, 21, 127-142.

Panda, S.K., S.S. Gedam, G. Rajaram, S. Sripathi, T.K. Pant and M.D. Rupesh (2014). A multi-technique study of the 29-31 October 2003 geomagnetic storm effect on low latitude ionosphere over Indian region with magnetometer, ionosonde, and GPS observations, Astrophysics and Space Science, 354, 267-274.

Rama Rao, P.V.S., S. Gopi Krishna, K. Niranjan and D.S.V.V.D. Prasad (2006). Temporal and spatial variations in TEC using simultaneous measurements from the Indian GPS network of receivers during the low solar activity period of 2004-2005, Annales Geophysicae, 24, 3279-3292.

Saito, A., S. Fukao and S. Mayazaki (1998). High resolution mapping of TEC perturbations with the GSI GPS network over Japan, Geophysical Research Letters, 25, 3079-3082.

Şentürk, E. (2014).Total Electron Content Computation On Carrier Wawes and Criterions Regarding To Calculation Quality (in Turkish), Master Thesis, Kocaeli University, Enstitute of Natural and Applied Science, Kocaeli, Turkey, 372703.

Sharma, K., R.S. Dabas and S. Ravindran (2012). Study of total electron content variations over equatorial and low latitude ionosphere during extreme solar minimum, Astrophysics and Space Science, 341, 277-286.

Weber, E.J., J.A. Klobuchar, J. Buchau, H.C. Carlson, R.C. Livingston, O. de la Beaujardiere, M. McCready, J.G. Moore and G.J. Bishop (1986). Polar cap F layer patches: structure and dynamics, Journal of Geophysical Research: Space Physics, 91, 2121-2129.

Wells, D.E., N. Beck, D. Delikaraoglou, A. Kleusberg, E.J. Krakiwsky, G. Lachapelle, R.B. Langley, M. Nakiboglu, K.P. Schwarz, J.M. Tranquilla and P. Vanicek (1986). Guide to GPS Positioning, Canadian GPS Associates, Fredericton, N.B., Canada.

Zirari, S., P. Canalda and F. Spies (2009). Geometric and Signal Strength Dilution of Precision (DoP) Wi-Fi, International Journal of Computer Science Issues, $3,35-44$.

\footnotetext{
${ }^{\star}$ Corresponding author: Murat Selim Çepni, University of Kocaeli, Department of Geomatic Engineering, Kocaeli, Turkey; email: muratselim.cepni@kocaeli.edu.tr. 